\title{
Analysis of Spatial Locality in Search Engines Using P2p Network
}

\author{
Anik Sengupta \\ Manoj Kr. Mohapatra \\ Souvik Sonar \\ Dept of Computer Science and Engg, Dept of Computer Science and Engg, Dept of Computer Science and Engg, \\ National Institute of Technology, National Institute of Technology, National Institute of Technology, \\ Durgapur, India \\ Durgapur, India \\ Durgapur, India
}

\begin{abstract}
Geographical locality in search results is of importance to the end user. However, it is a nontrivial problem. We propose P2P based Search Engine server architecture as a solution to the problem. The search result is fragmented into categories based on hop counts to hit. We map the system to a P2P file sharing network model based on probabilistic distribution and analyse a simulation model to assess the feasibility of implementing such a system.
\end{abstract}

The full text of the article is not available in the cache. Kindly refer the IJCA digital library at www.ijcaonline.org for the complete article. In case, you face problems while downloading the full-text, please send a mail to editor at editor@ijcaonline.org 